International Journal of Pure and Applied Mathematics

Volume 111 No. 2 2016, 249-272

ISSN: 1311-8080 (printed version); ISSN: 1314-3395 (on-line version)

url: http://www.ijpam.eu

doi: 10.12732 /ijpam.v111i2.10

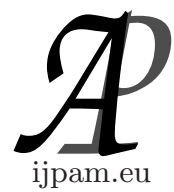

\title{
SOLUTION AND STABILITY OF TWO TYPES OF $n$-DIMENSIONAL QUARTIC FUNCTIONAL EQUATION IN GENERALIZED 2-NORMED SPACES
}

\author{
S. Murthy ${ }^{1}$, V. Govindhan ${ }^{2} \S$, M. Sree Shanmuga Velan ${ }^{3}$ \\ ${ }^{1}$ Department of Mathematics \\ Government Arts and Science College (For Men) \\ Krishnagiri, 635 001, Tamil Nadu, INDIA \\ ${ }^{2}$ Department of Mathematics \\ Sri Vidya Mandir Arts and Science College \\ Uthangarai, 636 902, Tamil Nadu, INDIA \\ ${ }^{3}$ Department of Mathematics \\ Hosur Institute of Technology and Science \\ Krishnagiri, 635 115, Tamil Nadu, INDIA
}

Abstract: In this paper, we investigate the general solution and generalized Ulam-Hyers stability of a $n$-dimensional quartic functional equation of the form

$$
\begin{gathered}
(8-n) f\left(\sum_{i=1}^{n} x_{i}\right)+\sum_{j=1}^{n} f\left(-x_{j}+\sum_{i=1 ; i \neq j} x_{i}\right)=4 \sum_{1 \leq i<j<k \leq n}\left(x_{i}+x_{j}+x_{k}\right)+(-4 n+14) \\
\sum_{i=1 ; i \neq j} f\left(x_{i}+x_{j}\right)+2 \sum_{i=1 ; i \neq j} f\left(x_{i}-x_{j}\right)+\sum_{j=1}^{n} f\left(2 x_{j}\right)+\left(2 n^{2}-14 n+14\right) \sum_{i=1}^{n} f\left(x_{i}\right),
\end{gathered}
$$

with $n \geq 3$ in generalized 2-normed space using two types of different methods.

AMS Subject Classification: 39B52, 39B82, 46 B99

Key Words: Ulam-Hyers stability, 2-normed space, quartic functional equation and fixed point

Received: $\quad$ August 31, 2016

Revised: $\quad$ October 7, 2016

Published: December 11, 2016

${ }^{\S}$ Correspondence author (c) 2016 Academic Publications, Ltd. url: www.acadpubl.eu 


\section{Introduction}

In 1940, before the guidance of the mathematics club of the University of Wisconsin S. M. Ulam [37] presented a list of unsolved problems. One of these problem can be considered as the starting point of a new line of investigations. The stability problem, suppose that a group $G$ and a metric group $H$ are given. For any $\epsilon>0$, does there exists a $\delta>0$ such that if a function $f: G \rightarrow H$ satisfies the inequality $d(f(x y), f(x) f(y))<\delta$ for all $x, y \in G$, then a homomorphism $a: G \rightarrow H$ exists with $d(f(x), a(x))<\epsilon$ for all $x \in G$ ? These kind of questions form the material of the stability theory [36]. In 1941, D. H. Hyers [15] answered the Ulam's problems for the case of approximately additive functions under the assumption that $G$ and $H$ are Banach spaces. Taking this fact into account, the additive functional equation

$$
f(x+y)=f(x)+f(y)
$$

is said to have Ulam-Hyers stability [14]. For more information see [1, 2, 3, 8, $9,10,11,12,13,16,28,32,34,38]$.

The U-type stability result for the quartic functional equation

$$
f(x+2 y)+f(x-2 y)+6 f(x)=4[f(x+y)+f(x-y)]+24 f(y)
$$

was first invented by J.M. Rassias in [27]. Subsequently, P.K. Sahoo and J.K. Chung [33], S.H. Lee et. al., [20] modified the J. M. Rassias equation (1.1) as

$$
f(2 x+y)+f(2 x-y)=4 f(x+y)+4 f(x-y)+24 f(x)-6 f(y) .
$$

Many other types of quartic functional equations were introduced and investigated in $[30,35]$.

Motivated by this observation, in this paper the authors investigate the general solution and generalized Ulam-Hyers stability of a $n$-dimensional quartic functional equation of the form,

$$
\begin{aligned}
(8-n) f\left(\sum_{i=1}^{n} x_{i}\right)+ & \sum_{j=1}^{n} f\left(-x_{j}+\sum_{i=1 ; i \neq j} x_{i}\right) \\
= & 4 \sum_{1 \leq i<j<k \leq n}\left(x_{i}+x_{j}+x_{k}\right)+(-4 n+14) \\
& \sum_{i=1 ; i \neq j} f\left(x_{i}+x_{j}\right)+2 \sum_{i=1 ; i \neq j} f\left(x_{i}-x_{j}\right)
\end{aligned}
$$




$$
+\sum_{j=1}^{n} f\left(2 x_{j}\right)+\left(2 n^{2}-14 n+14\right) \sum_{i=1}^{n} f\left(x_{i}\right)
$$

with $n \geq 3$ in generalized 2-normed space using two types of different methods.

\section{Preliminaries of 2-Normed Spaces}

Now, we present some basic definitions related to Generalized 2-normed spaces.

Definition 2.1. [24]. Let $X$ be a linear space. A function $N(.,$.$) :$ $X \times X \rightarrow[0, \infty)$ is called a generalized 2-normed space if it is satisfies the following:

$(G 2 N 1) N(x, y)=0$ if and only if $x$ and $y$ are linearly independent vectors.

$(G 2 N 2) \quad N(x, y)=N(y, x)$ for all $x, y \in X$.

$(G 2 N 3) \quad N(\lambda x, y)=|\lambda| N(x, y)$ for all $x, y \in X$ and $X=\phi, \phi$ is real or complex field.

$(G 2 N 4) \quad N(x+y, z) \leq N(x, z)+N(y, z)$; if for all $x, y, z \in X$.

The generalized 2-normed space is denoted by $(X, N(.,)$.$) .$

Definition 2.2. [24]. A sequence $\left\{x_{n}\right\}$ in a generalized 2-normed space $(X, N(.,)$.$) is called a Cauchy sequence if there exists two linearly independent$ elements $y$ and $z$ in $X$ such that $\{N(x n, y)\}$ and $\{N(x n, z)\}$ are real Cauchy sequences.

Definition 2.3. $[29,7]$. A sequence $\left\{x_{n}\right\}$ in a generalized 2-normed space $(X, N(.,)$.$) is called convergent if there exists x \in X$ such that

$$
\lim _{n \rightarrow \infty} N\left(x_{n}-x, y\right)=0
$$

then for all $y \in X$, we have

$$
\lim _{n \rightarrow \infty} N\left(x_{n}, y\right)=N(x, y)
$$

Definition 2.4. $[24,21]$. A generalized 2-normed space $(X, N(.,)$.$) is$ called generalized 2-Banach space if every Cauchy sequence is convergent. 


\section{A Solution of quartic functional equation}

It is well known $[3,4,5,6,17,18,19,22,23,25,26,31]$ that a function $f: X \rightarrow Y$ between a real vector spaces is quadratic if and only if there exists a unique symmetric biadditive function $B$ such that $f(x)=B(x, x)$ for all $x \in X$. The biadditive function $B$ is given by

$$
B(x, y)=\frac{1}{4}(f(x+y)-f(x-y)) .
$$

In this section, we prove that a function $f: X \rightarrow Y$ between the real vector spaces satisfies the functional equation (1.2) if and only if there exists a symmetric biquadratic function $F$ such that $f(x)=F(x, x)$ for all $x \in X$. Throughout this section $X$ and $Y$ will be real vector spaces.

Theorem 3.1. Let $X$ and $Y$ be a Real Vector Space, the mapping $f$ : $X \rightarrow Y$ satisfies the functional equation (1.2), for all $x, y \in X$ if and only if the mapping $f: X \rightarrow Y$ satisfies the functional equation (1.3) for all $x_{1}, x_{2}, \ldots, x_{n} \in$ $X$.

Proof. Assume that $f$ satisfies the functional equation (1.2). Putting $x=$ $y=0$ in (1.2), we have $f(0)=0$. Putting $x=0$ in (1.2) $f(y)=f(-y)$ for all $y \in X$. Putting $y=0$ and $y=x$ in (1.2), we obtain that

$$
f(2 x)=16 f(x),
$$

and for all $x \in X$, we have

$$
f(3 x)=81 f(x),
$$

respectively. Actually, we can lead to

$$
f(n x)=n^{4} f(x),
$$

for all $x \in X$ and all $n \in N$. Replacing $x$ and $y$ by $x_{1}+x_{2}$ and $x_{1}-x_{2}$ in (1.2), respectively, we get

$$
\begin{aligned}
f( & \left.2 x_{1}+2 x_{2}+x_{1}-x_{2}\right)+f\left(2 x_{1}+2 x_{2}-x_{1}+x_{2}\right) \\
= & 4 f\left(x_{1}+x_{2}+x_{1}-x_{2}\right)+4 f\left(x_{1}+x_{2}-x_{1}+x_{2}\right) \\
& +24 f\left(x_{1}+x_{2}\right)-6 f\left(x_{1}-x_{2}\right) f\left(3 x_{1}+x_{2}\right)+f\left(x_{1}+3 x_{2}\right) \\
= & 4 f\left(2 x_{1}\right)+4 f\left(2 x_{2}\right)+24 f\left(x_{1}+x_{2}\right)-6 f\left(x_{1}-x_{2}\right) f\left(3 x_{1}+x_{2}\right)+f\left(x_{1}+3 x_{2}\right) \\
= & 64 f\left(x_{1}\right)+64 f\left(x_{2}\right)+24 f\left(x_{1}+x_{2}\right)-6 f\left(x_{1}-x_{2}\right),
\end{aligned}
$$


for all $x_{1}, x_{2} \in X$. Replacing $x_{1}$ and $x_{2}$ by $x_{1}+x_{2}$ and $2 x_{2}$ in (1.2), respectively, we arrive to

$$
\begin{aligned}
f( & \left.2 x_{1}+2 x_{2}+2 x_{2}\right)+f\left(2 x_{1}+2 x_{2}-2 x_{2}\right) \\
= & 4 f\left(x_{1}+x_{2}+2 x_{2}\right)+4 f\left(x_{1}+x_{2}-2 x_{2}\right) \\
& \quad+24 f\left(x_{1}+x_{2}\right)-6 f\left(2 x_{2}\right) f\left(2\left(x_{1}+2 x_{2}\right)\right)+f\left(2 x_{1}\right) \\
= & 4 f\left(x_{1}+3 x_{2}\right)+4 f\left(x_{1}-x_{2}\right)+24 f\left(x_{1}+x_{2}\right)-6 f\left(2 x_{2}\right) 16 f\left(x_{1}+2 x_{2}\right)+16 f\left(x_{1}\right) \\
= & 4 f\left(x_{1}+3 x_{2}\right)+4 f\left(x_{1}-x_{2}\right)+24 f\left(x_{1}+x_{2}\right)-24 f\left(x_{2}\right) 4 f\left(x_{1}+2 x_{2}\right)+4 f\left(x_{1}\right) \\
= & f\left(x_{1}+3 x_{2}\right)+f\left(x_{1}-x_{2}\right)+6 f\left(x_{1}+x_{2}\right)-24 f\left(x_{2}\right),
\end{aligned}
$$

for all $x_{1}, x_{2} \in X$. Interchanging $x_{1}$ and $x_{2}$ in (3.2), we obtain that

$$
4 f\left(x_{2}+2 x_{1}\right)+4 f\left(x_{2}\right)=f\left(x_{2}+3 x_{1}\right)+f\left(x_{2}-x_{1}\right)+6 f\left(x_{2}+x_{1}\right)-24 f\left(x_{1}\right),
$$

for all $x_{1}, x_{2} \in X$. Adding (3.2) and (3.3) and also using (3.1), we obtain

$$
\begin{aligned}
4 f & \left(x_{1}+2 x_{2}\right)+4 f\left(x_{1}\right)+4 f\left(x_{2}+2 x_{1}\right)+4 f\left(x_{2}\right) \\
= & f\left(x_{1}+3 x_{2}\right)+f\left(x_{1}-x_{2}\right)+6 f\left(x_{1}+x_{2}\right)-24 f\left(x_{2}\right)+f\left(x_{2}+3 x_{1}\right) \\
& \quad+f\left(x_{2}-x_{1}\right)+6 f\left(x_{2}+x_{1}\right)-24 f\left(x_{1}\right) 4 f\left(x_{1}+2 x_{2}\right)+4 f\left(2 x_{1}+x_{2}\right) \\
= & f\left(x_{1}+3 x_{2}\right)+f\left(3 x_{1}+x_{2}\right)+12 f\left(x_{1}+x_{2}\right)+f\left(x_{1}-x_{2}\right) \\
& \quad+f\left(x_{2}-x_{1}\right)-24 f\left(x_{1}\right)-24 f\left(x_{2}\right)-4 f\left(x_{1}\right)-4 f\left(x_{2}\right) .
\end{aligned}
$$

Then using the result (3.1), we have

$$
\begin{aligned}
& \quad 4 f\left(x_{1}+2 x_{2}\right)+4 f\left(2 x_{1}+x_{2}\right)=64 f\left(x_{1}\right)+64 f\left(x_{2}\right)+24 f\left(x_{1}+x_{2}\right) \\
& -6 f\left(x_{1}-x_{2}\right)+12 f\left(x_{1}+x_{2}\right)+f\left(x_{1}-x_{2}\right)+f\left(x_{2}-x_{1}\right)-28 f\left(x_{1}\right)-28 f\left(x_{2}\right) .
\end{aligned}
$$

Using $f(-x)=f(x)$, we have

$$
\begin{aligned}
4 f\left(x_{1}+2 x_{2}\right)+4 f\left(2 x_{1}+x_{2}\right)= & 36 f\left(x_{1}\right)+36 f\left(x_{2}\right)+36 f\left(x_{1}+x_{2}\right)-6 f\left(x_{1}-x_{2}\right) \\
& +2 f\left(x_{1}-x_{2}\right) f\left(x_{1}+2 x_{2}\right)+f\left(2 x_{1}+x_{2}\right) \\
= & 9 f\left(x_{1}\right)+9 f\left(x_{2}\right)+9 f\left(x_{1}+x_{2}\right)-f\left(x_{1}-x_{2}\right)
\end{aligned}
$$

for all $x_{1}, x_{2} \in X$. Setting $x=x_{1} ; x_{2}=x_{3}$ in (1.2), we receive

$$
f\left(2 x_{1}+x_{3}\right)+f\left(2 x_{1}-x_{3}\right)=24 f\left(x_{1}\right)-6 f\left(x_{3}\right)+4 f\left(x_{1}+x_{3}\right)+4 f\left(x_{1}-x_{3}\right),
$$

for all $x_{1}, x_{3} \in X$. Replacing $x_{1}=x_{2}$ and $x_{2}=x_{3}$ in (1.2), we obtain

$$
f\left(2 x_{2}+x_{3}\right)+f\left(2 x_{2}-x_{3}\right)=24 f\left(x_{2}\right)-6 f\left(x_{3}\right)+4 f\left(x_{2}+x_{3}\right)+4 f\left(x_{2}-x_{3}\right),
$$


for all $x_{2}, x_{3} \in X$. Adding (3.5) and (3.6), that the resultant equation, we obtain

$$
\begin{aligned}
& 9 f\left(2 x_{1}+x_{3}\right)+9 f\left(2 x_{1}-x_{3}\right)+9 f\left(2 x_{2}+x_{3}\right)+9 f\left(2 x_{2}-x_{3}\right) \\
& =36 f\left(x_{1}+x_{3}\right)+36 f\left(x_{1}-x_{3}\right)+36 f\left(x_{2}+x_{3}\right) \\
& \quad+36 f\left(x_{2}-x_{3}\right)+216 f\left(x_{1}\right)+216 f\left(x_{2}\right)-108 f\left(x_{3}\right)
\end{aligned}
$$

for all $x_{1}, x_{2}, x_{3} \in X$. Replacing $x=2 x_{1}+x_{3}$ and $x_{2}=2 x_{2}+x_{3}$ in the equation (3.4), gives that

$$
\begin{aligned}
f( & \left.2 x_{1}+x_{3}+4 x_{2}+2 x_{3}\right)+f\left(4 x_{1}+2 x_{2}+2 x_{3}+x_{3}\right) \\
= & 9 f\left(2 x_{1}+x_{3}\right)+9 f\left(2 x_{2}+x_{3}\right)+9 f\left(2 x_{1}+x_{3}+2 x_{2}+x_{3}\right) \\
& -f\left(2 x_{1}+x_{3}-2 x_{2}-x_{3}\right) f\left(2 x_{1}+4 x_{2}+3 x_{3}\right)+f\left(4 x_{1}+2 x_{2}+3 x_{3}\right) \\
= & 9 f\left(2 x_{1}+x_{3}\right)+9 f\left(2 x_{2}+x_{3}\right)+9 f\left(2 x_{1}+2 x_{2}+2 x_{3}\right) \\
& -f\left(2 x_{1}-2 x_{2}\right) f\left(2 x_{1}+4 x_{2}+3 x_{3}\right)+f\left(4 x_{1}+2 x_{2}+3 x_{3}\right) \\
& -9 f\left(2 x_{1}+2 x_{2}+2 x_{3}\right)+f\left(2 x_{1}-2 x_{2}\right) \\
= & 9 f\left(2 x_{1}+x_{3}\right)+9 f\left(2 x_{2}+x_{3}\right) 9 f\left(2 x_{1}+x_{3}\right)+9 f\left(2 x_{2}+x_{3}\right) \\
= & f\left(2 x_{1}+4 x_{2}+3 x_{3}\right)+f\left(4 x_{1}+2 x_{2}+3 x_{3}\right) \\
& -9 f\left(2 x_{1}+2 x_{2}+2 x_{3}\right)+f\left(2 x_{1}-2 x_{2}\right)
\end{aligned}
$$

for all $x_{1}, x_{2}, x_{3} \in X$. Replacing $x_{1}$ and $x_{2}$ by $2 x_{1}-x_{3}$ and $x_{2}=2 x_{2}-x_{3}$ in the equation (3.4), we arrive that

$$
\begin{aligned}
f( & \left.2 x_{1}-x_{3}+4 x_{2}-2 x_{3}\right)+f\left(4 x_{1}-2 x_{3}+2 x_{2}-x_{3}\right) \\
= & 9 f\left(2 x_{1}-x_{3}\right)+9 f\left(2 x_{2}-x_{3}\right)+9 f\left(2 x_{1}-x_{3}+2 x_{2}-x_{3}\right) \\
& \quad-f\left(2 x_{2}-x_{3}-2 x_{2}+x_{3}\right) f\left(2 x_{1}+4 x_{2}-3 x_{3}\right)+f\left(4 x_{1}+2 x_{2}-3 x_{3}\right) \\
= & 9 f\left(2 x_{1}-x_{3}\right)+9 f\left(2 x_{2}-x_{3}\right)+9 f\left(2 x_{1}+2 x_{2}-2 x_{3}\right) \\
& \quad-f\left(2 x_{1}-2 x_{2}\right) f\left(2 x_{1}+4 x_{2}-3 x_{3}\right)+f\left(4 x_{1}+2 x_{2}-3 x_{3}\right) \\
& \quad-9 f\left(2 x_{1}+2 x_{2}-2 x_{3}\right)+f\left(2 x_{1}-2 x_{2}\right) \\
= & 9 f\left(2 x_{1}-x_{3}\right)+9 f\left(2 x_{2}-x_{3}\right) 9 f\left(2 x_{1}-x_{3}\right)+9 f\left(2 x_{2}-x_{3}\right) \\
= & f\left(2 x_{1}+4 x_{2}-3 x_{3}\right)+f\left(4 x_{1}+2 x_{2}-3 x_{3}\right) \\
- & 9 f\left(2 x_{1}+2 x_{2}-2 x_{3}\right)+f\left(2 x_{1}-2 x_{2}\right)
\end{aligned}
$$

for all $x_{1}, x_{2}, x_{3} \in X$. Adding (3.8) and (3.9), and using the result (1.2), we obtain

$$
\begin{aligned}
9 f( & \left.2 x_{1}+x_{3}\right)+9 f\left(2 x_{2}+x_{3}\right)+9 f\left(2 x_{1}-x_{3}\right)+9 f\left(2 x_{2}-x_{3}\right) \\
= & 4 f\left(x_{1}+2 x_{2}+3 x_{3}\right)+4 f\left(x_{1}+2 x_{2}-3 x_{3}\right)+24 f\left(x_{1}+2 x_{2}\right) \\
& -6 f\left(3 x_{3}\right)+4 f\left(2 x_{1}+x_{2}+3 x_{3}\right)+4 f\left(2 x_{1}+x_{2}-3 x_{3}\right) \\
& +24 f\left(2 x_{1}+x_{2}\right)-6 f\left(3 x_{3}\right)-144 f\left(x_{1}+x_{2}+x_{3}\right) \\
& -144 f\left(x_{1}+x_{2}-x_{3}\right)+32 f\left(x_{1}-x_{2}\right),
\end{aligned}
$$


for all $x_{1}, x_{2}, x_{3} \in X$. By the equations (3.7) and (3.10), and rearranging the equation, we obtain

$$
\begin{gathered}
36 f\left(x_{1}+x_{3}\right)+36 f\left(x_{1}-x_{3}\right)+216 f\left(x_{1}\right)+54 f\left(x_{3}\right)+36 f\left(x_{2}+x_{3}\right)+36 f\left(x_{2}-x_{3}\right) \\
+216 f\left(x_{2}\right)-54 f\left(x_{3}\right)=4 f\left(x_{1}+2 x_{2}+3 x_{3}\right)+4 f\left(x_{1}+2 x_{2}-3 x_{3}\right)+24 f\left(x_{1}+2 x_{2}\right) \\
-6 f\left(3 x_{3}\right)+4 f\left(2 x_{1}+x_{2}+3 x_{3}\right)+4 f\left(2 x_{1}+x_{2}-3 x_{3}\right)+24 f\left(2 x_{1}+x_{2}\right) \\
-6 f\left(3 x_{3}\right)-144 f\left(x_{1}+x_{2}+x_{3}\right)-144 f\left(x_{1}+x_{2}-x_{3}\right)+32 f\left(x_{1}-x_{2}\right)
\end{gathered}
$$

for all $x_{1}, x_{2}, x_{3} \in X$. Replacing $x_{1}$ and $x_{2}$ by $2 x_{1}+x_{3}$ and $x_{2}=2 x_{2}-x_{3}$ in the equation (3.4), we arrive that

$$
\begin{aligned}
& f\left(2 x_{1}-x_{3}+4 x_{2}-2 x_{3}\right)+f\left(4 x_{1}+2 x_{3}+2 x_{2}-x_{3}\right) \\
& =9 f\left(2 x_{1}+x_{3}\right)+9 f\left(2 x_{2}-x_{3}\right)+9 f\left(2 x_{1}+x_{3}+2 x_{2}-x_{3}\right) \\
& \quad-f\left(2 x_{1}+x_{3}-2 x_{2}+x_{3}\right) f\left(2 x_{1}+4 x_{2}-x_{3}\right)+f\left(4 x_{1}+2 x_{2}+x_{3}\right) \\
& \quad=9 f\left(2 x_{1}+x_{3}\right)+9 f\left(2 x_{2}-x_{3}\right)+9 f\left(2 x_{1}+2 x_{2}\right)-f\left(2 x_{1}-2 x_{2}+2 x_{3}\right)
\end{aligned}
$$

which implies that,

$$
\begin{aligned}
9 f\left(2 x_{1}+x_{3}\right)+9 f\left(2 x_{2}-x_{3}\right)= & f\left(2 x_{1}+4 x_{2}-x_{3}\right)+f\left(4 x_{1}+2 x_{2}+x_{3}\right) \\
& -9 f\left(2 x_{1}+2 x_{2}\right)+f\left(2 x_{1}-2 x_{2}+2 x_{3}\right)
\end{aligned}
$$

for all $x_{1}, x_{2}, x_{3} \in X$. Replacing $x_{1}=2 x_{1}-x_{3}$ and $x_{2}=2 x_{2}+x_{3}$ in the equation (3.4), we obtain that

$$
\begin{aligned}
& f\left(2 x_{1}-x_{3}+4 x_{2}+2 x_{3}\right)+f\left(4 x_{1}-2 x_{3}+2 x_{2}+x_{3}\right) \\
& =9 f\left(2 x_{1}-x_{3}\right)+9 f\left(2 x_{2}+x_{3}\right)+9 f\left(2 x_{1}-x_{3}+2 x_{2}+x_{3}\right) \\
& \quad-f\left(2 x_{1}-x_{3}-2 x_{2}-x_{3}\right) f\left(2 x_{1}+4 x_{2}+x_{3}\right)+f\left(4 x_{1}+2 x_{2}-x_{3}\right) \\
& \quad=9 f\left(2 x_{1}-x_{3}\right)+9 f\left(2 x_{2}+x_{3}\right)+9 f\left(2 x_{1}+2 x_{2}\right)-f\left(2 x_{1}-2 x_{2}-2 x_{3}\right)
\end{aligned}
$$

which gives that,

$$
\begin{aligned}
9 f\left(2 x_{1}-x_{3}\right)+9 f\left(2 x_{2}+x_{3}\right)= & f\left(2 x_{1}+4 x_{2}+x_{3}\right)+f\left(4 x_{1}+2 x_{2}-x_{3}\right) \\
& -9 f\left(2 x_{1}+2 x_{2}\right)+f\left(2 x_{1}-2 x_{2}-2 x_{3}\right)
\end{aligned}
$$

for all $x_{1}, x_{2}, x_{3} \in X$. Adding (3.12) and (3.13), we have,

$$
\begin{aligned}
& 9 f\left(2 x_{1}+x_{3}\right)+9 f\left(2 x_{2}-x_{3}\right)+9 f\left(2 x_{1}-x_{3}\right)+9 f\left(2 x_{2}+x_{3}\right) \\
& =f\left(2 x_{1}+4 x_{2}-x_{3}\right)+f\left(4 x_{1}+2 x_{2}+x_{3}\right)-9 f\left(2 x_{1}+2 x_{2}\right)+f\left(2 x_{1}-2 x_{2}+2 x_{3}\right) \\
& \quad+f\left(2 x_{1}+4 x_{2}+x_{3}\right)+f\left(4 x_{1}+2 x_{2}-x_{3}\right)-9 f\left(2 x_{1}+2 x_{2}\right)+f\left(2 x_{1}-2 x_{2}-2 x_{3}\right)
\end{aligned}
$$


for all $x_{1}, x_{2}, x_{3} \in X$. Using the equation (1.2) in the above equation, we get

$$
\begin{aligned}
9 f & \left(2 x_{1}+x_{3}\right)+9 f\left(2 x_{2}-x_{3}\right)+9 f\left(2 x_{1}-x_{3}\right)+9 f\left(2 x_{2}+x_{3}\right) \\
= & 4 f\left(x_{1}+2 x_{2}+x_{3}\right)+4 f\left(x_{1}+2 x_{2}-x_{3}\right)+24 f\left(x_{1}+2 x_{2}\right)-6 f\left(x_{3}\right) \\
& +4 f\left(2 x_{1}+x_{2}+x_{3}\right)+4 f\left(2 x_{1}+x_{2}-x_{3}\right)+24 f\left(2 x_{1}+x_{2}\right)-6 f\left(x_{3}\right) \\
& \quad-288 f\left(x_{1}+x_{2}\right)+16 f\left(x_{1}-x_{2}+x_{3}\right)+16 f\left(x_{1}-x_{2}-x_{3}\right)
\end{aligned}
$$

for all $x_{1}, x_{2}, x_{3} \in X$. The above equation implies that

$$
\begin{aligned}
9 f & \left(2 x_{1}+x_{3}\right)+9 f\left(2 x_{2}-x_{3}\right)+9 f\left(2 x_{1}-x_{3}\right)+9 f\left(2 x_{2}+x_{3}\right) \\
= & 4 f\left(x_{1}+2 x_{2}+x_{3}\right)+4 f\left(x_{1}+2 x_{2}-x_{3}\right)+24 f\left(x_{1}+2 x_{2}\right)-6 f\left(x_{3}\right) \\
& \quad+4 f\left(2 x_{1}+x_{2}+x_{3}\right)+4 f\left(2 x_{1}+x_{2}-x_{3}\right)+24 f\left(2 x_{1}+x_{2}\right)-6 f\left(x_{3}\right) \\
& \quad-288 f\left(x_{1}+x_{2}\right)+16 f\left(x_{1}-x_{2}+x_{3}\right)+16 f\left(x_{1}-x_{2}-x_{3}\right)
\end{aligned}
$$

for all $x_{1}, x_{2}, x_{3} \in X$. Replacing $x_{3}$ by $3 x_{3}$ in the above equation (3.16), we arrive that

$$
\begin{aligned}
9 f( & \left.2 x_{1}+3 x_{3}\right)+9 f\left(2 x_{2}-3 x_{3}\right)+9 f\left(2 x_{1}-3 x_{3}\right)+9 f\left(2 x_{2}+3 x_{3}\right) \\
= & 4 f\left(x_{1}+2 x_{2}+3 x_{3}\right)+4 f\left(x_{1}+2 x_{2}-3 x_{3}\right)+24 f\left(x_{1}+2 x_{2}\right)-6 f\left(3 x_{3}\right) \\
& +4 f\left(2 x_{1}+x_{2}+3 x_{3}\right)+4 f\left(2 x_{1}+x_{2}-3 x_{3}\right)+24 f\left(2 x_{1}+x_{2}\right)-6 f\left(3 x_{3}\right) \\
& -288 f\left(x_{1}+x_{2}\right)+16 f\left(x_{1}-x_{2}+3 x_{3}\right)+16 f\left(x_{1}-x_{2}-3 x_{3}\right)
\end{aligned}
$$

for all $x_{1}, x_{2}, x_{3} \in X$. Using the equation (3.11), from the resultant equation, we obtain

$$
\begin{aligned}
= & 4 f\left(x_{1}+2 x_{2}+3 x_{3}\right)+4 f\left(x_{1}+2 x_{2}-3 x_{3}\right)+24 f\left(x_{1}+2 x_{2}\right)-6 f\left(3 x_{3}\right) \\
& +4 f\left(2 x_{1}+x_{2}+3 x_{3}\right)+4 f\left(2 x_{1}+x_{2}-3 x_{3}\right)+24 f\left(2 x_{1}+x_{2}\right)-6 f\left(3 x_{3}\right)
\end{aligned}
$$

for all $x_{1}, x_{2}, x_{3} \in X$. Substitute (3.18) in (3.17), we get

$$
\begin{aligned}
& 9 f\left(2 x_{1}+x_{3}\right)+9 f\left(2 x_{2}-x_{3}\right)+9 f\left(2 x_{1}-x_{3}\right)+9 f\left(2 x_{2}+x_{3}\right) \\
& =36 f\left(x_{1}+x_{3}\right)+36 f\left(x_{1}-x_{3}\right)+216 f\left(x_{1}\right)-54 f\left(x_{3}\right)+36 f\left(x_{2}+x_{3}\right) \\
& \quad+36 f\left(x_{2}-x_{3}\right)+216 f\left(x_{2}\right)-54 f\left(x_{3}\right)+144 f\left(x_{1}+x_{2}+x_{3}\right)+144 f\left(x_{1}+x_{2}-x_{3}\right) \\
& \quad-32 f\left(x_{1}-x_{2}\right)-288 f\left(x_{1}+x_{2}\right)+16 f\left(x_{1}-x_{2}+3 x_{3}\right)+16 f\left(x_{1}-x_{2}-3 x_{3}\right)
\end{aligned}
$$


for all $x_{1}, x_{2}, x_{3} \in X$. Replacing $x_{1}=x_{1}-x_{2}+3 x_{3}$ and $x_{2}=x_{1}-x_{2}-3 x_{3}$ in (3.4), which implies that

$$
\begin{aligned}
f( & \left.3 x_{1}-3 x_{2}-3 x_{3}\right)+f\left(3 x_{1}-3 x_{2}+3 x_{3}\right) \\
= & 9 f\left(x_{1}-x_{2}+3 x_{3}\right)+9 f\left(x_{1}-x_{2}-3 x_{3}\right)+9 f\left(2 x_{1}-2 x_{3}\right) \\
& \quad-f\left(6 x_{3}\right) 9 f\left(x_{1}-x_{2}+3 x_{3}\right)+9 f\left(x_{1}-x_{2}-3 x_{3}\right) \\
= & f\left(3 x_{1}-3 x_{2}-3 x_{3}\right)+f\left(3 x_{1}-3 x_{2}+3 x_{3}\right)-9 f\left(2 x_{1}-2 x_{3}\right) \\
& \quad+f\left(6 x_{3}\right) 9 f\left(x_{1}-x_{2}+3 x_{3}\right)+9 f\left(x_{1}-x_{2}-3 x_{3}\right) \\
= & 81 f\left(x_{1}-x_{2}-x_{3}\right)+81 f\left(x_{1}-x_{2}+x_{3}\right)-144 f\left(x_{1}-x_{3}\right)+1296 f\left(x_{3}\right)
\end{aligned}
$$

for all $x_{1}, x_{2}, x_{3} \in X$. Divided by $\left(\frac{16}{9}\right)$, we obtain

$$
\begin{aligned}
& 16 f\left(x_{1}-x_{2}+3 x_{3}\right)+16 f\left(x_{1}-x_{2}-3 x_{3}\right) \\
& \quad=144 f\left(x_{1}-x_{2}-x_{3}\right)+144 f\left(x_{1}-x_{2}+x_{3}\right)-256 f\left(x_{1}-x_{3}\right)+2304 f\left(x_{3}\right)
\end{aligned}
$$

for all $x_{1}, x_{2}, x_{3} \in X$. using the equation (3.19), (3.20) and (3.21), we will have

$$
\begin{aligned}
9 f( & \left.2 x_{1}+3 x_{3}\right)+9 f\left(2 x_{2}-3 x_{3}\right)+9 f\left(2 x_{1}-3 x_{3}\right)+9 f\left(2 x_{2}+3 x_{3}\right) \\
= & 36 f\left(x_{1}+x_{3}\right)+36 f\left(x_{1}-x_{3}\right)+216 f\left(x_{1}\right)-54 f\left(x_{3}\right)+36 f\left(x_{2}+x_{3}\right) \\
& +36 f\left(x_{2}-x_{3}\right)+216 f\left(x_{2}\right)-54 f\left(x_{3}\right)+144 f\left(x_{1}+x_{2}+x_{3}\right) \\
& \quad+144 f\left(x_{1}+x_{2}-x_{3}\right)-32 f\left(x_{1}-x_{2}\right)-288 f\left(x_{1}+x_{2}\right) \\
& +144 f\left(x_{1}-x_{2}-x_{3}\right)+144 f\left(x_{1}-x_{2}+x_{3}\right)-256 f\left(x_{1}-x_{2}\right)+2304 f\left(x_{3}\right)
\end{aligned}
$$

for all $x_{1}, x_{2}, x_{3} \in X$. Setting $x_{1}=2 x_{1}+3 x_{3}$ and $x_{2}=2 x_{1}-3 x_{3}$ in (3.4), which gives that

$$
\begin{aligned}
& f\left(2 x_{1}+3 x_{3}+4 x_{1}-6 x_{3}\right) f\left(4 x_{1}+6 x_{3}+2 x_{1}-3 x_{3}\right) \\
& =9 f\left(2 x_{1}+3 x_{3}\right)+9 f\left(2 x_{1}-3 x_{3}\right)+9 f\left(2 x_{1}+3 x_{3}+2 x_{1}-3 x_{3}\right) \\
& \quad-f\left(2 x_{1}+3 x_{3}-2 x_{1}-3 x_{3}\right) f\left(6 x_{1}-3 x_{3}\right)+f\left(6 x_{1}+3 x_{3}\right) \\
& =9 f\left(2 x_{1}+3 x_{3}\right)-9 f\left(2 x_{1}-3 x_{3}\right)+9 f\left(4 x_{1}\right)-f\left(6 x_{3}\right)
\end{aligned}
$$

for all $x_{1}, x_{2}, x_{3} \in X$. Replacing $x_{1}=2 x_{2}-3 x_{3}$ and $x_{2}=2 x_{2}+3 x_{3}$ in (3.4), we arrive that

$$
\begin{aligned}
f\left(6 x_{2}+3 x_{3}\right)+ & f\left(6 x_{2}-3 x_{3}\right) \\
& =9 f\left(2 x_{2}-3 x_{3}\right)-9 f\left(2 x_{2}+3 x_{3}\right)+9 f\left(4 x_{2}\right)-f\left(6 x_{3}\right)
\end{aligned}
$$


for all $x_{1}, x_{2}, x_{3} \in X$. Adding (3.23) and (3.24), we obtain

$$
\begin{gathered}
9 f\left(2 x_{1}+3 x_{3}\right)+9 f\left(2 x_{1}-3 x_{3}\right)+9 f\left(2 x_{2}+3 x_{3}\right)+9 f\left(2 x_{2}-3 x_{3}\right) \\
+9 f\left(2 x_{2}+3 x_{3}\right)=324 f\left(x_{1}+x_{3}\right)+324 f\left(x_{1}-x_{3}\right)+1944 f\left(x_{1}\right) \\
\quad-486 f\left(x_{3}\right)+324 f\left(x_{2}+x_{3}\right)+324 f\left(x_{2}-x_{3}\right)+1944 f\left(x_{2}\right) \\
-486 f\left(x_{3}\right)-2304 f\left(x_{1}\right)-2304 f\left(x_{2}\right)+2592 f\left(x_{3}\right)
\end{gathered}
$$

for all $x_{1}, x_{2}, x_{3} \in X$. From equation (3.22) and (3.25), L. H. S are equal, that

$$
\begin{aligned}
& 36 f\left(x_{1}+x_{3}\right)+36 f\left(x_{1}-x_{3}\right)+216 f\left(x_{1}\right)-54 f\left(x_{3}\right)+36 f\left(x_{2}+x_{3}\right)+36 f\left(x_{2}-x_{3}\right) \\
& +216 f\left(x_{2}\right)-54 f\left(x_{3}\right)+144 f\left(x_{1}+x_{2}+x_{3}\right)+144 f\left(x_{1}+x_{2}-x_{3}\right) \\
& -32 f\left(x_{1}-x_{2}\right)-288 f\left(x_{1}+x_{2}\right)+144 f\left(x_{1}-x_{2}-x_{3}\right)+144 f\left(x_{1}-x_{2}+x_{3}\right) \\
& -256 f\left(x_{1}-x_{2}\right)+2304 f\left(x_{3}\right)=324 f\left(x_{1}+x_{3}\right)+324 f\left(x_{1}-x_{3}\right) \\
& \quad+1944 f\left(x_{1}\right)-486 f\left(x_{3}\right)+324 f\left(x_{2}+x_{3}\right)+324 f\left(x_{2}-x_{3}\right) \\
& \quad+1944 f\left(x_{2}\right)-486 f\left(x_{3}\right)-2304 f\left(x_{1}\right)-2304 f\left(x_{2}\right)+2592 f\left(x_{3}\right)
\end{aligned}
$$

for all $x_{1}, x_{2}, x_{3} \in X$. From the resultant equation (3.26), we get

$$
\begin{aligned}
144 & {\left[f\left(x_{1}+x_{2}+x_{3}\right)+f\left(x_{1}+x_{2}-x_{3}\right)+f\left(x_{1}-x_{2}-x_{3}\right)+f\left(x_{1}-x_{2}+x_{3}\right)\right] } \\
= & 324 f\left(x_{1}+x_{3}\right)+324 f\left(x_{1}-x_{3}\right)+1944 f\left(x_{1}\right)-486 f\left(x_{3}\right) \\
& +324 f\left(x_{2}+x_{3}\right)+324 f\left(x_{2}-x_{3}\right)+1944 f\left(x_{2}\right)-486 f\left(x_{3}\right)-2304 f\left(x_{1}\right) \\
& -2304 f\left(x_{2}\right)+2592 f\left(x_{3}\right) 36 f\left(x_{1}+x_{3}\right)+36 f\left(x_{1}-x_{3}\right)+216 f\left(x_{1}\right) \\
& -54 f\left(x_{3}\right)+36 f\left(x_{2}+x_{3}\right)+36 f\left(x_{2}-x_{3}\right)+216 f\left(x_{2}\right)-54 f\left(x_{3}\right) \\
& -32 f\left(x_{1}-x_{2}\right)-288 f\left(x_{1}+x_{2}\right)-256 f\left(x_{1}-x_{2}\right)+2304 f\left(x_{3}\right)
\end{aligned}
$$

which implies that

$$
\begin{aligned}
& f\left(x_{1}+x_{2}+x_{3}\right)+f\left(x_{1}+x_{2}-x_{3}\right)+f\left(x_{1}-x_{2}-x_{3}\right)+f\left(x_{1}-x_{2}+x_{3}\right) \\
& =2\left(f\left(x_{1}+x_{2}\right)+f\left(x_{1}+x_{3}\right)+f\left(x_{2}+x_{3}\right)\right) \\
& \quad+2\left(f\left(x_{1}-x_{2}\right)+f\left(x_{1}-x_{3}\right)+f\left(x_{2}-x_{3}\right)\right)-4\left\{f\left(x_{1}\right)+f\left(x_{2}\right)+f\left(x_{3}\right)\right\}
\end{aligned}
$$

for all $x_{1}, x_{2}, x_{3} \in X$. From the equation (3.27), rearranging we get

$$
\begin{aligned}
& f\left(x_{1}+x_{2}+x_{3}\right)+f\left(-x_{1}+x_{2}-x_{3}\right)+f\left(x_{1}+x_{2}-x_{3}\right)+f\left(x_{1}-x_{2}+x_{3}\right) \\
& =\left(4 \sum_{1 \leq i<j<k \leq 3} f\left(x_{i}+x_{j}+x_{k}\right)\right)^{+}+(-4 \times 3+14) \sum_{1 \leq i<j \leq 3} f\left(x_{i}+x_{j}\right) \\
& \quad+2 \sum_{1 \leq i<j \leq 3} f\left(x_{i}-x_{j}\right) \sum_{j=1}^{3} f\left(2 x_{j}\right)+\left(2 \times 3^{2}-14 \times 3+4\right) \sum_{i=1}^{3} f\left(x_{i}\right)
\end{aligned}
$$


for all $x_{1}, x_{2}, x_{3} \in X$. Similarly one can easily verify that this result is true for four variables, we get

$$
\begin{aligned}
& f\left(x_{1}+x_{2}+x_{3}+x_{4}\right)+f\left(-x_{1}+x_{2}-x_{3}+x_{4}\right)+f\left(x_{1}+x_{2}-x_{3}+x_{4}\right) \\
& +f\left(x_{1}-x_{2}+x_{3}+x_{4}\right)=\left(4 \sum_{1 \leq i<j<k \leq 4} f\left(x_{i}+x_{j}+x_{k}\right)\right) \\
& \quad+(-4 \times 4+14) \sum_{1 \leq i<j \leq 4} f\left(x_{i}+x_{j}\right) \\
& \quad+2 \sum_{1 \leq i<j \leq 4} f\left(x_{i}-x_{j}\right) \sum_{j=1}^{4} f\left(2 x_{j}\right)+\left(2 \times 4^{2}-14 \times 4+4\right) \sum_{i=1}^{4} f\left(x_{i}\right)
\end{aligned}
$$

for all $x_{1}, x_{2}, x_{3} \in X$. Similarly one can easily verify that this result is true for $n$ variables, Extending this result, for any positive integer $n$, we arrive

$$
\begin{aligned}
& f\left(\sum_{i=1}^{n} x_{i}\right)+\sum_{j=1}^{n} f\left(-x_{j}+\sum_{i=1 ; i \neq j} x_{i}\right)=4 \sum_{1 \leq i<j<k \leq n}\left(x_{i}+x_{j}+x_{k}\right)+(-4 n+14) \\
& \sum_{i=1 ; i \neq j} f\left(x_{i}+x_{j}\right)+2 \sum_{i=1 ; i \neq j} f\left(x_{i}-x_{j}\right)+\sum_{j=1}^{n} f\left(2 x_{j}\right)+\left(2 n^{2}-14 n+14\right) \sum_{i=1}^{n} f\left(x_{i}\right)
\end{aligned}
$$

for all $x_{1}, x_{2}, x_{3} \in X$. This completes the proof of the first part of the theorem.

Conversely, assume that $f: X \rightarrow Y$ satisfies the functional equation (3.29). Now we prove that the function $f: X \rightarrow Y$ satisfies the functional equation (1.2). Now replacing $\left(x_{1}, x_{2}, x_{3}\right)$ by $(x, x, y)$ in three variables that

$$
\begin{aligned}
(8- & n) f(2 x+y)+f(2 x-y)+2 f(y) \\
= & 4 f(2 x+y)+2(-4 n+14) f(x+y)+(2.2) f(x-y) \\
& +4 f(y)(8-n) f(2 x+y)+f(2 x-y)+2 f(y) \\
= & 4 f(2 x+y)+2(-4 n+14) f(x+y)+(2.2) f(x-y) \\
& +(2.2) f(x-y)+(-4 n+14) f(2 x)+2 .\left(2 n^{2}-14 n+14\right) f(x) \\
& +\left(2 n^{2}-14 n+14\right) f(y)+(-4 n+14) f(2 x)+f(2 x)+f(2 y)
\end{aligned}
$$

for all $x, y \in X$. And again from the equation (3.29) for four variables, which 
gives that

$$
\begin{aligned}
(8- & n) f(2 x+y)+f(2 x+y)+f(2 x-y)+2 f(y) \\
= & 4 f(2 x+y)+2(-4 n+14) f(x+y)+(2.4) f(x-y)+(2.2) f(x-y) \\
& +2(-4 n+14) f(x)+(-4 n+14) f(2 x)+2 .\left(2 n^{2}-14 n+14\right) f(x) \\
& +\left(2 n^{2}-14 n+14\right) f(y)+2(-4 n+14) f(x)+(-4 n+14) f(2 x) \\
& +(-4 n+14) f(y)+f(2 x)+f(2 y)+(2.2) f(2 x)+2 f(2 y)
\end{aligned}
$$

for all $x, y \in X$. And again from the equation (3.29) for five variables, gives

$$
\begin{aligned}
(8- & n) f(2 x+y)+f(2 x-y)+2 f(2 x+y)+2 f(y) \\
= & 4 f(2 x+y)+(4.4) f(x+y)+2(-4 n+14) f(x+y) \\
& +(2.4) f(x-y)+(2.2) f(x-y)+4(-4 n+14) f(x)+(2.4) f(x) \\
& +(1.4) f(y)+(-4 n+14) f(2 x)+2 .\left(2 n^{2}-14 n+14\right) f(x) \\
& +\left(2 n^{2}-14 n+14\right) f(y)+4(-4 n+14) f(x)+2(-4 n+14) f(y) \\
& +(-4 n+14) f(2 x)+f(2 x)+f(2 y)+(2.4) f(2 x)+(2.2) f(2 y)
\end{aligned}
$$

for all $x, y \in X$. Combining the equations (3.30), (3.31) and (3.32) and for $n$ variables, gives that

$$
\begin{aligned}
(8- & n) f(2 x+y)+(n-3) f(2 x+y)+f(2 x-y)+2 f(y) \\
= & 4 f(2 x+y)+2(n-3)[4 f(x+y)]+2[2 f(x-y)]+(-4 n+14)[2 f(x+y)] \\
& +2(n-3)(-4 n+14) f(x)+(n-3)(n-4)[4 f(x)]+\frac{(n-3)(n-4)}{2}[4 f(y)] \\
& +(-4 n+14) f(2 x)+2 .\left(2 n^{2}-14 n+4\right) f(x)+\left(2 n^{2}-14 n+14\right) f(y) \\
& +(n-3)(-4 n+14) f(y)+4(n-3) f(2 x)+(2.2) f(2 y) \\
& +(4 n-12) f(x)+2 n f(y)-6 f(y)+f(2 x)+f(2 y)+f(2 x)
\end{aligned}
$$

for all $x, y \in X$. Now from the equation (3.33), and rearranging that

$$
\begin{aligned}
8 f( & 2 x+y)+3 f(2 x+y)+f(2 x-y)+2 f(y) \\
= & 4 f(2 x+y)+(2 n-6)[4 f(x+y)]+4 f(x-y)+(-8 n+28) f(x+y) \\
& +(2 n-6)(-4 n+14) f(x)+\left(n^{2}-7 n-12\right)[4 f(x)] \\
& +\left(n^{2}-7 n-12\right)[2 f(y)]+(-4 n+14) f(2 x)+\left(4 n^{2}-28 n+8\right) f(x) \\
& +\left(2 n^{2}-14 n+4\right) f(y)+\left(n^{2}-7 n-42\right) f(y)+(4 n-12) f(2 x) \\
& +(4 n-12) f(x)+2 n f(2 y)+-6 f(y)+16 f(x)+16 f(y)+16 f(x)
\end{aligned}
$$


for all $x, y \in X$. Now from the equation (3.34), modified as

$$
\begin{aligned}
5 f(2 x+y)+f(2 x-y)+2 f(y)= & 4 f(2 x+y)+8 n f(x+y)-24 f(x+y) \\
& +4 f(x-y)-8 n f(x+y)+28 f(x+y)+ \\
& -8 n^{2} f(x)+52 f(x)-84 f(x)+4 n^{2} f(x) \\
& -28 n f(x)-48 f(x)+2 n^{2} f(y)-14 n f(y) \\
& -24 f(y)-4 n f(2 x)+14 f(2 x)+4 n^{2} f(x) \\
& -28 n f(x)-8 f(x)+2 n^{2} f(y)-14 n f(y) \\
& +4 f(y)+n^{2} f(y)-7 n f(y)-42 f(y)+4 n f(2 x) \\
& -12 f(2 x)+4 n f(x)-12 f(x)+2 n f(2 y) \\
& +-6 f(y)+16 f(x)+16 f(y)+16 f(x)
\end{aligned}
$$

$$
\begin{aligned}
& \begin{array}{r}
5 f(2 x+y)+f(2 x-y)+2 f(y) \\
=4 f(2 x+y)+4 f(x+y)+4 f(x-y)+24 f(x)-4 f(y), \\
5 f(2 x+y)-4 f(2 x+y)+f(2 x-y) \\
=-2 f(y)+4 f(x+y)+4 f(x-y)+24 f(x)-4 f(y),
\end{array}
\end{aligned}
$$

which implies that,

$$
f(2 x+y)+f(2 x-y)=4 f(x+y)+4 f(x-y)+24 f(x)-6 f(y)
$$

for all $x, y \in X$. Setting $y$ by $-y$ in the equation (3.36), we obtain

$$
f(2 x-y)+f(2 x+y)=4 f(x-y)+4 f(x+y)+24 f(x)-6 f(-y)
$$

for all $x, y \in X$. Using the even function then we have from (3.37), we obtain that

$$
f(2 x-y)+f(2 x+y)=4 f(x-y)+4 f(x+y)+24 f(x)-6 f(y)
$$

for all $x, y \in X$. Adding the equations (3.36) and (3.38), we get

$$
2 f(2 x-y)+2 f(2 x+y)=8 f(x-y)+8 f(x+y)+24 f(x)-6 f(y)
$$

Dividing the above by 2 , we arrive that

$$
f(2 x+y)+f(2 x-y)=4 f(x+y)+4 f(x-y)+24 f(x)-6 f(y)
$$

for all $x, y \in X$. Which completes the proof of the Theorem. 


\section{Stability of $n$-Dimensional Quartic Functional Equation (1.3)}

In this section, let us consider $X$ be a generalized 2-normed space and $Y$ be a generalized 2-Banach space respectively. Define a mapping $Q_{f}: X^{n} \rightarrow Y$ by

$$
\begin{gathered}
Q_{f}(x)=Q_{f}\left(x_{1}, x_{2}, x_{3}, \ldots, x_{n}\right)=(8-n) f\left(\sum_{i=1}^{n} x_{i}\right)+\sum_{j=1}^{n} f\left(-x_{j}+\sum_{i=1 ; i \neq j} x_{i}\right) \\
-4 \sum_{1 \leq i<j<k \leq n}\left(x_{i}+x_{j}+x_{k}\right)-(-4 n+14) \sum_{i=1 ; i \neq j} f\left(x_{i}+x_{j}\right)-2 \sum_{i=1 ; i \neq j} f\left(x_{i}-x_{j}\right) \\
-\sum_{j=1}^{n} f\left(2 x_{j}\right)-\left(2 n^{2}-14 n+14\right) \sum_{i=1}^{n} f\left(x_{i}\right)
\end{gathered}
$$

for all $x_{1}, x_{2}, x_{3}, \ldots, x_{n} \in X^{n}$ and $n \geq 3$.

Theorem 4.1. Let $\psi: X^{n} \rightarrow[0, \infty)$ be a function such that

$$
\sum_{m=0}^{\infty} \frac{\psi\left(\left(m^{n j} x_{1}, z\right),\left(m^{n j} x_{2}, z\right)\right),\left(m^{n j} x_{3}, z\right), \ldots,\left(m^{n j} x_{n}, z\right)}{m^{4 n j}}
$$

converges and

$$
\lim _{n \rightarrow \infty}\left\{\frac{\psi\left(\left(m^{n j} x_{1}, z\right),\left(m^{n j} x_{2}, z\right),\left(m^{n j} x_{3}, z\right), \ldots,\left(m^{n j} x_{n}, z\right)\right)}{m^{4 n j}}\right\}=0
$$

for all $x_{1}, x_{2}, x_{3}, \ldots, x_{n} \in X^{n}, z \in X$ and let $f: X \rightarrow Y$ be a function satisfying the inequality

$$
N\left(Q_{f}(x), z\right) \leq \psi\left(\left(x_{1}, z\right),\left(x_{2}, z\right), \ldots,\left(x_{n}, z\right)\right)
$$

for all $x_{1}, x_{2}, x_{3}, \ldots, x_{n} \in X^{n}, z \in X$. Then there exists a unique quartic function $F: X \rightarrow Y$ such that

$$
N(f(x)-F(x), z) \leq \sum_{n=\frac{1-j}{2}}^{\infty} \frac{\varphi\left(2^{n j} x, z\right)}{16^{n j}}
$$

where $\varphi\left(2^{n j} x, z\right)=\psi(x, 0,0, \ldots, z)$ for all $x, z \in X$. The function $F(x)$ is defined by

$$
N(F(x), z)=\lim _{n \rightarrow \infty} N\left(\frac{f\left(2^{n j} x\right)}{16^{n j}}, z\right)
$$

for all $x, z \in X$ and $j= \pm 1$. 
Proof. Assume that $j=1$. Replacing $\left(x_{1}, x_{2}, x_{3}, \ldots, x_{n}\right)$ by $(x, 0,0, \ldots 0)$ in (4.2), we get

$$
\begin{gathered}
N\left((8-n) f(x)+f(-x)+(n-1) f(x)-2\left[n^{2}+3 n+2\right] f(x)-(-4 n+14)\right. \\
\left.(n-1) f(x)-2(n-1) f(x)-\left(2 n^{2}-14 n+14\right) f(x), z\right) \leq \psi(x, 0,0, \ldots, 0) \\
N\left(\frac{f(2 x)}{16}-f(x), z\right) \leq \frac{\psi(x, 0,0, \ldots, 0)}{16}
\end{gathered}
$$

for all $x, z \in X$. Letting

$$
\varphi(x, z)=\frac{\psi(x, 0,0, \ldots, 0)}{16}
$$

in the equation (4.5), we arrive

$$
N\left(\frac{f(2 x)}{16}-f(x), z\right) \leq \varphi(x, z)
$$

for all $x, z \in X$. Now setting $x$ by $2 x$ and dividing by 16 in (4.6), we obtain

$$
N\left(\frac{f\left(2^{2} x\right)}{16^{2}}-\frac{f(2 x)}{16}, z\right) \leq \varphi(2 x, z)
$$

for all $x, z \in X$. Using the inequality (4.6) and (4.7), and the definition of (G2N4), we obtain

$$
\begin{aligned}
& N\left(\frac{f\left(2^{2} x\right)}{16^{2}}-f(x), z\right)=N\left(\frac{f\left(2^{2} x\right)}{16^{2}}-\frac{f(2 x)}{16}+\frac{f(2 x)}{16}-f(x), z\right) \\
& \leq N\left(\frac{f\left(2^{2} x\right)}{16^{2}}-\frac{f(2 x)}{16}, z\right)+N\left(\frac{f(2 x)}{16}-f(x), z\right) \\
& \leq \varphi(x, z)+\frac{\varphi(2 x, z)}{16}
\end{aligned}
$$

for all $x, z \in X$. In general for any positive integer $m$, we have

$$
N\left(\frac{f\left(2^{m} x\right)}{16^{m}}-f(x), z\right) \leq \sum_{n=0}^{m-1} \frac{\varphi\left(2^{n} x, z\right)}{16^{n}} \leq \sum_{n=0}^{\infty} \frac{\varphi\left(2^{n} x, z\right)}{16^{n}}
$$


for all $x, z \in X$. In order to prove the convergence of the sequence $\left\{\frac{f\left(2^{n} x, z\right)}{16^{n}}\right\}$ replace $x$ by $2^{l} x$ and dividing by $16^{l}$ in (4.9), for any $n, l \geq 0$, we obtain

$$
\begin{gathered}
N\left(\frac{f\left(2^{l+n} x\right)}{16^{l+n}}-\frac{f\left(2^{l} x\right)}{16^{l}}, z\right) \leq \frac{1}{16^{l}} N\left(\frac{f\left(2^{l} .2^{n} x\right)}{16^{n}}-f\left(2^{l} x\right), z\right) \\
\leq \sum_{n=0}^{\infty} \frac{\varphi\left(2^{l+n} x, z\right)}{16^{l+n}}=0 \text { as } \rightarrow \infty .
\end{gathered}
$$

for all $x, z \in X$. Also, we have

$$
\begin{gathered}
N\left(\frac{f\left(2^{l+n} x\right)}{16^{l+n}}-\frac{f\left(2^{l} x\right)}{16^{l}}, z_{1}\right) \leq \frac{1}{16^{l}} N\left(\frac{f\left(2^{l} \cdot 2^{n} x\right)}{16^{n}}-f\left(2^{l} x\right), z_{1}\right) \\
\leq \sum_{n=0}^{\infty} \frac{\varphi\left(2^{l+n} x, z_{1}\right)}{16^{l+n}}=0 \text { as } \rightarrow \infty
\end{gathered}
$$

for all $x, z \in X$. Hence there exists two linearly independent elements $z$ and $z_{1}$ in $X$ such that $\left\{\frac{f\left(2^{n} x\right)}{16^{n}}, z\right\}$ and $\left\{\frac{f\left(2^{n} x\right)}{16^{n}}, z_{1}\right\}$ are real Cauchy sequences. Hence the sequence $\left\{\frac{f\left(2^{n} x\right)}{16^{n}}\right\}$ is Cauchy sequence, since $Y$ is complete, there exists a mapping $F: X \rightarrow Y$ such that

$$
N(F(x), z)=\lim _{n \rightarrow \infty} N\left(\frac{f\left(2^{n} x\right)}{16^{n}}, z\right)
$$

for all $x, z \in X$. Letting $n \rightarrow \infty$ in (4.9), we see that (4.4) holds for all $x, z \in X$. To prove $F$ satisfies (4.4), setting $\left(x_{1}, x_{2}, \ldots, x_{n}\right)$ by $\left(2^{n} x_{1}, 2^{n} x_{2}, 2^{n} x_{3}, \ldots, 2^{n} x_{n}\right)$ and dividing by $16^{n}$ in (4.2) and allow $n \rightarrow \infty$, we arrive

$$
N\left(D_{F}\left(x_{1}, x_{2}, \ldots, x_{n}\right), z\right)=0 .
$$

Hence $F$ satisfies (4.3) for all $x_{1}, x_{2}, \ldots, x_{n}, z \in X$. To prove $F$ is unique. Let $J(x)$ be another quartic mapping satisfying (4.4) and (4.3). Then

$$
\begin{gathered}
N(F(x)-J(x), z)=\frac{1}{16^{n}} N\left(F\left(2^{n} x\right)-J\left(2^{n} x\right), z\right) \\
\leq \frac{1}{16^{n}}\left\{N\left(F\left(2^{n} x\right)-f\left(2^{n} x\right), z\right)+N\left(f\left(2^{n} x\right)-J\left(2^{n} x\right), z\right)\right\} \\
\leq \sum_{n=0}^{\infty} \frac{\varphi\left(2^{l+n} x, z\right)}{16^{l+n}}=0 \text { asl } \rightarrow \infty .
\end{gathered}
$$

The right hand side of the above inequality converges to 0 as $n \rightarrow \infty$ for all $x, z \in X$. Hence $F$ is Unique, for $j=-1$, we can prove the similar type pf stability result. This completes the proof of the Theorem. 
The following corollary is an immediate consequence of Theorem ?? concerning the stability of (4.4).

Corollary 4.2. Let $\delta$ and $s$ be the non-negative real numbers. If a function $f: X \rightarrow Y$ satisfying the inequality

$$
N\left(Q_{f}(x), z\right) \leq\left\{\begin{array}{l}
\delta \\
\delta \sum_{k=1}^{n}\left\|x_{k}, z\right\|^{s}, \quad s>4(\text { or }) s<4 \\
\delta\left\{\sum_{k=1}^{n}\left\|x_{k}, z\right\|^{n s}+\prod_{k=1}^{n}\left\|x_{k}, z\right\|^{n s}\right\}, s>\frac{4}{n}(\text { or }) s<\frac{4}{n}
\end{array}\right.
$$

for all $x_{1}, x_{2}, \ldots, x_{n}, z \in X$. Then there exists a unique quartic function $F$ : $X \rightarrow Y$ such that

$$
N(f(x)-F(x), z) \leq\left\{\begin{array}{l}
\frac{16 \delta}{15}, \\
\frac{16 \delta}{\left|16-2^{s}\right|}\|x, z\|^{s} \\
\frac{16 \delta}{\left|16-2^{n s}\right|}\|x, z\|^{n s}
\end{array}\right.
$$

for all $x \in X$ and $z \in X$.

\section{Stability of $n$-Dimensional Quartic Functional Equation (1.3): Fixed Point Method}

The following Theorem provide the stability result of (1.3) in fixed point method. For proving the stability result, we define the following, $m_{i}$ is a constant such that

$$
m_{i}=\left\{\begin{array}{l}
2, \text { if } i=0 \\
\frac{1}{2} \text { if } i=1
\end{array}\right.
$$

and $\eta$ is the set such that

$$
\eta=\{g / g: X \rightarrow Y ; g(0)=0\}
$$

Theorem 5.1. Let $f: X \rightarrow Y$ be a mapping for which there exists a function $\psi: X^{n} \rightarrow(0, \infty)$ with the condition

$$
\sum_{m=0}^{\infty} \frac{\psi\left(\left(m_{i}^{n} x_{1}, z\right),\left(m_{i}^{n} x_{2}, z\right)\right),\left(m_{i}^{n} x_{3}, z\right), \ldots,\left(m_{i}^{n} x_{n}, z\right)}{m_{i}^{4 n}}
$$


converges and

$$
\lim _{n \rightarrow \infty}\left\{\frac{\psi\left(\left(m_{i}^{n} x_{1}, z\right),\left(m_{i}^{n} x_{2}, z\right),\left(m_{i}^{n} x_{3}, z\right), \ldots,\left(m_{i}^{n} x_{n}, z\right)\right)}{m_{i}^{4 n}}\right\}=0
$$

for all $x_{1}, x_{2}, x_{3}, \ldots, x_{n} \in X, z \in X$ satisfying the functional inequality

$$
N\left(Q_{f}(x), z\right) \leq \psi\left(\left(x_{1}, z\right),\left(x_{2}, z\right), \ldots,\left(x_{n}, z\right)\right)
$$

for all $x_{1}, x_{2}, x_{3}, \ldots, x_{n} \in X, z \in X$. If there exists $L=L(i)<1$ such that the function

$$
x \rightarrow \varphi(x, z)=16 \varphi\left(\left(\frac{x}{2}, z\right),(0, z),(0, z), \ldots,(0, z)\right)
$$

has the property, that for all $x \in X$,

$$
\frac{\varphi\left(m_{i} x, z\right)}{m_{i}^{4}}=L \varphi(x, z)
$$

Then there exists a unique quartic function $F: X \rightarrow Y$ satisfying the functional equation (4.3) and for all $x \in X$, we have

$$
N(f(x)-F(x), z) \leq \frac{L^{1-i}}{1-L} \varphi(x, z) .
$$

Proof. Let $\phi$ be a general metric on $\eta$ such that

$$
d(g, h)=\inf \{k \in(0, \infty): N(g(x)-h(x), z) \leq k \phi(x, z), x, z \in X\}
$$

It is easy to see that $(\eta, d)$ is complete. Define $T: \eta \rightarrow \eta$ by

$$
T_{f}(x)=\frac{1}{m_{i}^{4}} f\left(m_{i} x\right)
$$

for all $x \in X$. For $f, h \in \eta$, we have $d(f, h)=k$.

$$
\begin{gathered}
N(f(x)-h(x), z) k \varphi x, z \\
N\left(\frac{f\left(m_{i} x\right)}{m_{i}^{4}}-\frac{h\left(m_{i} x\right)}{m_{i}^{4}}, z\right) \leq \frac{1}{m_{i}^{4}} k \varphi\left(m_{i} x, z\right)
\end{gathered}
$$

which implies that

$$
d\left(T_{f}(x), T_{h}(x)\right) \leq K L .
$$


Hence, $d(T g, T h) \leq L d(f, h)$, for all $f, h \in \eta$. Therefore, $T$ is strictly contractive mapping on $\eta$ with Lipschitz constant $L$. Replacing $\left(x_{1}, x_{2}, \ldots, x_{n}\right)$ by $(x, 0,0, \ldots, 0)$ in $(5.3)$ and using the definition of $\varphi(x, z)$, we get

$$
N(f(2 x)-16 f(x), z) \leq \varphi(2 x, z)
$$

for all $x, z \in X$. Applying the equation (5.4) in the above equation for $i=0$, we have

$$
N(f(2 x)-16 f(x), z) \leq \varphi(2 x, z)
$$

which implies that

$$
N\left(T_{f}(x)-f(x)\right) \leq L \varphi(x, z)
$$

for all $x, z \in X$. Hence, we arrive

$$
N\left(T_{f}(x), f(x)\right) \leq L=L^{1-i}
$$

for all $x \in X$. Replacing $x$ by $\frac{x}{2}$ in (5.6) and using the definition of $\varphi(x)$ for $i=1$, we have

$$
\|f(2 x)-16 f(x / 2)\|_{P} \leq \varphi(x)
$$

which gives that

$$
\left\|f(2 x)-T_{f}(x)\right\|_{P} \leq \varphi(x)
$$

for all $x \in X$. Hence we arrive that

$$
N\left(T_{f}(x), f(x)\right) \leq 1=L^{1-i}
$$

for all $x \in X$. Then from (5.7) and (5.8) we can conclude that

$$
N\left(T_{f}(x), f(x)\right) \leq L^{1-i}<\infty
$$

for all $x \in X$. Now from the fixed point alternative in both cases, it follows that there exists a fixed point $F$ of $T$ in $\eta$ such that

$$
N(F(x), z)=\lim _{m \rightarrow \infty} N\left(\frac{\left(m_{i}^{n} x, z\right)}{m_{i}^{4 n}}, z\right)
$$

for all $x \in X$. To prove $F$ satisfies the functional equation (4.4), we can use the same idea in Theorem 4.1. Since $F$ is unique fixed point of $T$ in the set

$$
\zeta=\{f \in \eta: d(f, F)\}
$$

therefore, $F$ is unique function such that for all $x, z \in X$, we have

$$
N(f(x)-F(x), z) \leq K \varphi(x, z) .
$$


Again using the fixed point alternative, we obtain

$$
d(f, F) \leq \frac{1}{1-L} d\left(f, T_{f}\right) \leq \frac{L^{1-i}}{1-L}
$$

gives that

$$
N(f(x)-F(x), z) \leq \frac{L^{1-i}}{1-L} \varphi(x, z) .
$$

This completes the proof of the Theorem.

The following corollary is an immediate consequence of Theorem 5.1 concerning the stability of (4.4).

Corollary 5.2. Let $f: X \rightarrow Y$ be a mapping there exists a real numbers $\Omega$ and $s$ such that

$$
N\left(Q_{f}(x), z\right) \leq\left\{\begin{array}{l}
\Omega, \\
\Omega \sum_{k=1}^{n}\left\|x_{k}, z\right\|^{s}, \quad s>2(\text { or }) s<2 \\
\Omega\left\{\sum_{k=1}^{n}\left\|x_{k}, z\right\|^{n s}+\prod_{k=1}^{n}\left\|x_{k}, z\right\|^{n s}\right\}, s>\frac{2}{n}(\text { or }) s<\frac{2}{n}
\end{array}\right.
$$

for all $x_{1}, x_{2}, \ldots, x_{n}, z \in X$. Then there exists a unique quartic function $F$ : $X \rightarrow Y$ such that

$$
N(f(x)-F(x), z) \leq\left\{\begin{array}{l}
\frac{16|\Omega|}{15}, \\
\frac{16|\Omega|}{\left|16-2^{s}\right|}\|x, z\|^{s}, \\
\frac{16|\Omega|}{\left|16-2^{n s}\right|}\|x, z\|^{n s}
\end{array}\right.
$$

for all $x \in X$.

Proof. Set $\varphi(x, z)$ as right hand side of the equation (5.12), for all $x_{1}, x_{2}, x_{3}$, $\ldots, x_{n} \in X$. It is easy to conclude that

$$
\frac{1}{m_{i}^{4}} \varphi\left(m_{i}^{4} x\right) \rightarrow 0 \text { as } n \rightarrow \infty .
$$

Thus (5.1) holds. But we have

$$
\varphi(x, z)=16 \psi\left(\left(\frac{x}{2}, z\right),(0, z),(0, z), \ldots,(0, z)\right)
$$


Now

$$
\frac{1}{m_{i}^{4}} \varphi\left(m_{i} x, z\right)=\left\{\begin{array}{l}
16 m_{i}^{-4} \Omega, \\
m_{i}^{s-4} 2^{4-s}\|x, z\|^{s}, \\
m_{i}^{n s-4} 2^{4-n s}\|x, z\|^{n s},
\end{array}=L \varphi(x)\right.
$$

where, $L=m_{i}^{-4}, L=m_{i}^{s-4}$ and $L=m_{i}^{n s-4}$ for the respective assumption of $\psi$. Hence the inequality (5.4) holds. Now, from (5.5), we prove the following cases for conditions. Case: (i). $L=2^{-4}$, if $i=0$, we have

$$
\begin{gathered}
N(f(x)-F(x), z) \leq \frac{L^{1-0}}{1-L} \varphi(x, z)=\frac{\left(2^{-4}\right)^{1-0}}{1-2^{-4}} 16 \Omega \\
N(f(x)-F(x), z) \leq \frac{16}{15} \Omega .
\end{gathered}
$$

Case: (ii). $L=2^{-4}$ and if $i=1$

$$
\begin{gathered}
N(f(x)-F(x), z) \leq \frac{L^{1-1}}{1-L} \varphi(x, z)=\frac{\left(\left(\frac{1}{2}\right)^{-4}\right)^{1-1}}{1-\left(\frac{1}{2}\right)^{-4}} 16 \Omega \\
N(f(x)-F(x), z) \leq \frac{-16}{15} \Omega .
\end{gathered}
$$

Case: (iii). $L=2^{s-4}$ and if $i=0$

$$
\begin{gathered}
N(f(x)-F(x), z) \leq \frac{2^{s-4}}{1-2^{s-4}} \Omega 2^{4-s}\|x, z\|^{s}=\frac{2^{s-4}}{1-2^{s} \cdot 2^{-4}} \Omega 2^{4-s}\|x, z\|^{s} \\
N(f(x)-F(x), z) \leq \frac{16}{16-2^{s}} \Omega\|x, z\|^{s}
\end{gathered}
$$

Case: (iv). $L=\frac{1}{2^{s-4}}$ and if $i=1$

$$
\begin{gathered}
N(f(x)-F(x), z) \leq \frac{L^{1-1}}{1-L} \varphi(x, z)=\frac{\left(\left(\frac{1}{2}\right)^{s-4}\right)^{1-1}}{1-2^{4-s}} \Omega 2^{4-s}\|x, z\|^{s} \\
N(f(x)-F(x), z) \leq \frac{-16}{16-2^{s}} \Omega\|x, z\|^{s}
\end{gathered}
$$

Case: (v). $L=2^{n s-4}$ and if $i=0$

$$
N(f(x)-F(x), z) \leq \frac{L^{1-0}}{1-L} \varphi(x, z)
$$




$$
\begin{gathered}
=\frac{2^{n s-4}}{1-2^{n s-4}} \Omega 2^{4-n s}\|x, z\|^{n} s \\
=\frac{2^{n s-4}}{1-2^{n s} \cdot 2^{-4}} \Omega 2^{4-n s}\|x, z\|^{n s} \\
N(f(x)-F(x), z) \leq \frac{16}{16-2^{n s}} \Omega\|x, z\|^{n s}
\end{gathered}
$$

Case: (vi). $L=\left(\frac{1}{2}\right)^{n s-4}$ and if $i=1$

$$
\begin{gathered}
N(f(x)-F(x), z) \leq \frac{L^{1-1}}{1-L} \varphi(x, z) \\
=\frac{1}{1-2^{-n s+4}} \Omega 2^{4-n s}\|x, z\|^{n} s \\
=\frac{1}{1-2^{-n s} \cdot 2^{4}} \Omega 2^{4-n s}\|x, z\|^{n s} \\
N(f(x)-F(x), z) \leq-\frac{16}{16-2^{n s}} \Omega\|x, z\|^{n s} .
\end{gathered}
$$

\section{References}

[1] J. Aczel, J. Dhomberes, Functional Equations in several variables, Cambridge university press (1989).

[2] T. Aoki, On the stability of linear transformation in Banach spaces, J. Math. Soc. Japan, 2 (1950), 64-66.

[3] M. Arunkumar, S. Murthy, G. Ganapathy, Solution and Stability of $n$-dimensional Quadratic functional equation, ICCMSC, 2012 Computer and Information science (CIS), springer verlag-Germany, vol. 238 (2012), 384-394.

[4] M. Arunkumar, S. Murthy, V. Govindan, General solution and generalized Ulam-Hyers stability of a generalized n-type additive quadratic functional equation in Banach spaces and Banach Algebra: Using direct and fixed point method, International journal of Advanced Mathematical sciences, 3(1) (2015) 25-64.

[5] M. Arunkumar, S. Murthy, V. Govindan and T. Namachivayam, General solution and Four types of Ulam-Hyers stability of n-dimensional additive functional equation in Banach and fuzzy Banach spaces: Hyers direct and Fixed point methods, International Journal of applied Engineering and research, 324-338.

[6] M. Arunkumar, K. Ravi, M. J. Rassias, Stability of a Quartic and orthogonally Quartic functional equation, Bulltien of Mathematical Analysis and application, 3(3), 2011, 13-24.

[7] M. Arunkumar, S. Murthy, G. Ganapathy, Stability of a functional equation having nth order solution in Generalized 2-normed spaces, International Journal of mathematical sciences and Eng. Applications, vol. 5, No. IV, (July 2011) 361-369. 
[8] T. Bag and S. K. Samanta, Finite dimensional fuzzy normed linear spaces, J. fuzzy Math. $11(3)$ (2003), 687-705.

[9] D.G. Bourgin, Classes of Transformations and bordering transformations, Bull. Amer. Math. Soc. Calcutta. Soc. 57, (1951), 223-237.

[10] S. C. Chang, J. N. Mordeson, Fuzzy linear operator and fuzzy normed linear spaces, Bull. Calcutta Soc. 86 (1994), 429-436.

[11] S. Czerwik, Fundamental equations and Inequalities in several variables, World Scientific, River edge, NJ, 2002.

[12] C. Czerwik, On the stability of the Quadratic mapping in normed spaces, Abh.Math. Sem. univ. Hamburg, 62 (1992), 59-64.

[13] P. Guvruta, A Generalization of the Hyers-Ulam-Rassias stability of approximately additive mappings, J. Math. Anal. Appl., 184 (1994), 431-436.

[14] D. H. Hyers, On the stability of linear functional equations, Proc. Natl. Acad. Sci. USA, 27 (1941), 222-224.

[15] D. H. Hyers, G. Issac. Th. M. Rassias, Stability of functional equations in several variables, Birkhauser, Basel, 1998.

[16] S. M. Jung, Hyers-Ulam-Rassias stability of functional equations, Mathematical Analysis, Hadronic press, palm harbor, 2001.

[17] S. S. Jin and Y. H. Lee, Fuzzy stability of a general quadratic functional equation deriving from quadratic and additive mappings, Abstract Appl. Anal., 2011 (2011), Article ID 534120, 15 pages.

[18] S. S. Jin and Y. H. Lee, Fuzzy stability of a general quadratic functional equation, Adv. Fuzzy syst., 2011 (2011), Article ID 791695, 9 pages.

[19] S. S. Jin and Y. H. Lee, Fuzzy stability of the Cauchy additive and quadratic type functional equation, Common korean math. Soc. 27 (2012), 523-535.

[20] S. H. Lee, S. M. Im and I. S. Hwang, quadratic functional equations, J. Math. Anal. Appl., 307 (2005) 387-394.

[21] S. S. Kim, Y. J. Cho and A. White, Linear operators on linear 2-normed spaces, G lasnik Math. 27 (42) (1992), 63-70.

[22] S. Murthy, M. Arunkumar, G. Ganapathy, Solution and stability of n-dimensional cubic functional equation in F-spaces: Direct and Fixed point methods, Proceeding of the international conferences of Mathematical Sciences (Elsevier India), 81-88.

[23] S. Murthy, M. Arunkumar, V. Govindhan, General solution and generalized Hyers-Ulam stability of n-dimensional quadratic functional equation in instuitionistic fuzzy Normed spaces: using direct and fixed point methods, Global journal of pure and applied Mathematics (Submitted).

[24] Z. Lewandowska, Linear operators on a generalized 2-normed spaces, Bull. Math. Soc. Sci., Math, Roumanie, 42 (90) No. 4 (1999), 353-368.

[25] A. Najati, On the stability of quartic functional equation, J. Math. Anal. App. 340 (1), (2008), 569-574.

[26] A. Najati, The generalized Hyers-Ulam stability of a cubic functional equation, Turk. J. math., 31 (2007), 1-14. 
[27] J. M. Rassias, On approximately of approximately linear mappings of linear mappings, J. Functional Anal., USA, 46 (1982), 126-130.

[28] Th. M. Rassias, On the stability of the linear mapping in Banach spaces, Proc. Amer. Math. Soc. 72, (1978), No. 2, 297-300.

[29] F. Raymond W and C. Goel Je., Geometry of linear 2-Normed spaces, Nova Sciences publishers, New York, 2001.

[30] K. Ravi and M. Arunkumar, Hyers-Ulam-Rassias stability of a Quartic functional Equation, International Journal of Pure and Applied mathematics, Vol. 34, No. 2, 2007, $247-260$.

[31] K. Ravi and M. Arunkumar, On a General solution of a Quartic functional equation, J. of Combinatorics Information and system sciences, Vol. 33, No. 1, 3-4 (2008), 373-386.

[32] A. M. Robert, A course in P-adic analysis, springer-verlag, New York (2000).

[33] P. K. Sahoo and J. K. Chung, On the general solution of a quartic functional equation, Bull. Korean. Math. Soc. 40 (4) (2003), 565-576.

[34] F. Skof, Local properties and approximations of operators, Rend. Sem. math. Fis. Milano 53 (1983), 113-129.

[35] E. Thandapani, K. Ravi, and M. Arunkumar, On the solution of the generalized Quartic functional equation, Far East Journal of Applied Mathematics, Vol. 24, No. 3, 2006, 297-312.

[36] S. M. Ulam, A Collection of Mathematical Problems, Interscience Publ., New York, 1960.

[37] S. M. Ulam, Problem in Modern mathematics, Sciences editions, Joha Wiley and sons, Inc., New York (1969).

[38] A. White, 2-Banach Spaces, Math. Nachr., 42 (1969), 43-60. 